\title{
Cluster-based Aggregate Forecasting for Residential Electricity Demand using Smart Meter Data
}

\author{
Tri Kurniawan Wijaya, Matteo Vasirani, Samuel Humeau, Karl Aberer \\ School of Computer and Communication Sciences \\ EPFL, Switzerland \\ Email: \{tri-kurniawan.wijaya, matteo.vasirani, samuel.humeau, karl.aberer\}@epfl.ch
}

\begin{abstract}
While electricity demand forecasting literature has focused on large, industrial, and national demand, this paper focuses on short-term (1 and 24 hour ahead) electricity demand forecasting for residential customers at the individual and aggregate level. Since electricity consumption behavior may vary between households, we first build a feature universe, and then apply Correlation-based Feature Selection to select features relevant to each household. Additionally, smart meter data can be used to obtain aggregate forecasts with higher accuracy using the so-called Cluster-based Aggregate Forecasting (CBAF) strategy, i.e., by first clustering the households, forecasting the clusters' energy consumption separately, and finally aggregating the forecasts. We found that the improvement provided by CBAF depends not only on the number of clusters, but also more importantly on the size of the customer base.
\end{abstract}

\section{INTRODUCTION}

The exploitation of renewable energy, the integration of distributed energy resources at the distribution level, and the electrification of private transportation are considered as suitable governmental policies to tackle some of the problems of advanced societies, such as reducing $\mathrm{CO}_{2}$ emissions or increasing energy efficiency [11]. In recent years, these solution concepts started to pose new challenges to the existing power grids, whose hierarchical, centrally-controlled structure has remained unchanged for a century. For example, the exploitation of renewable sources such as solar or wind may be problematic due to their variable and intermittent nature [38], while the integration of distributed energy resources may cause congestion and atypical power flows that threaten system's reliability [27]. On the other hand, as a part of smart grid initiatives, smart meters have been widely deployed to understand the energy consumption behavior of the demand side. More specifically, it contains the information of how end users consume electricity in near real time. However, this also means that utility companies worldwide face challenges on managing big (smart meter) data on their hands of at least big volume, big velocity, and big value, whose benefits are waiting to be discovered [39].

In this context, energy consumption prediction for different time horizons (e.g., 1 hour ahead, 1 day ahead, 1 month ahead) and space scales (e.g., distribution transformer, individual house-level meter) is also becoming crucial for many applications, such as frequency and voltage regulation, demand response (to estimate customer's baseline [37]), and autonomous energy and emergency management [28], [40]. While longterm load forecasting (1-10 years ahead) is important for

\footnotetext{
A preliminary version of this work has appeared in [19].
}

planning both, transmission and distribution networks, shortterm load forecasting (hours to days ahead) is important for the demand response, online scheduling, and security functions of an energy management system. In this paper, we use the terms energy consumption (or demand) and load interchangeably.

Many techniques for energy consumption prediction have been inspired by research on statistical and machine learning, from Linear Regression [16], [30], ARMA [18], [34], and Generalized Additive Models [4], [10], [41] to Neural Networks [3], [15], [23] and Support Vector Regression [9], [32]. However, these techniques have been typically used at very large space scales, such as predicting the electrical load of a market segment serving thousands of customers or even an entire country.

In this paper, we focus on forecasting electricity consumption of residential customers, leveraging smart meter data. Since energy consumption behavior might vary among households, a feature that are relevant for one house might not be relevant for others. Additionally, we consider a large number of houses. Thus, feature selection has to be done automatically. To this end, we first build a (large) feature universe, and then automatically determine the relevant features for each house using the Correlation-based Feature Selection [14], which selects subset of features set that are highly correlated with the response variable while having low inter-correlation between each other (see Section III-A).

Next, we found that smart meter data can be used to provide an aggregate forecast with higher accuracy, compared to traditional methods, using the so-called Cluster-based Aggregate Forecasting (or CBAF for short) by first clustering the households, forecasting the clusters separatelty, and finally, aggregating the forecasts. We find that the improvement provided by the CBAF strategy (compared to the traditional aggregate forecasts) depends not only on the number of the clusters, but more importantly on the size of the customer base. That is, the larger the customer base, the higher the improvement (see Section IV-B). Thus, our finding offers additional insight to the practitioners who wish to implement this strategy in the real world.

The paper is organized as follows. In Section II we describe the dataset and evaluation metrics. In Section III we present the features, methods, and experimental evaluations on forecasting energy consumption of a large number of households at the individual level. In Section IV we present insights gained from CBAF. In Section V we review related works in the area, and finally, we conclude in Section VI. 


\section{Dataset and Evaluation Metrics}

\section{A. Dataset}

We use the detailed data underlying electricity consumption behaviour provided in anonymized format by the Commission for Energy Regulation (CER) in Ireland. ${ }^{1}$ This dataset is the result of the Electricity Customer Behaviour Trials (CBTs), which took place during 2009 and 2010 with over 5,000 Irish homes and businesses participating. The participants in the trials had an electricity smart meter installed in their homes/premises, which collected energy consumption measurements every half hour. The objective of the trial was evaluating the impact that different Time-Of-Use (TOU) tariffs have on the consumption behaviour.

Although the CER has carefully cleaned the data (e.g., multiple imputation for the missing values-see [35] Appendix 2 ), there are still a small number of missing values found in the dataset. In this work, unless stated otherwise, we choose customers who have no missing values in their measurements. Furthermore, to avoid bias due to the TOU tariffs, we consider only the residential households in the control group of the trial, i.e.,, those customers with a flat rate that did not change their consumption behavior in response to a TOU tariff. This results in the selection of 782 customers. The measurements are aggregated into hourly timeslots. For all results presented in this paper, we use the first year (from July 2009 to June 2010) as the training set, and the remaining 6 months (from July 2010 to December 2010) as the test set.

\section{B. Evaluation metrics}

In the literature, there are three widely used metrics to evaluate the accuracy of a forecasting algorithm: the Mean Absolute Percentage Error (MAPE), the Mean Absolute Error (MAE), and the Root Mean Square Error (RMSE). Given a time series $S=\left\{s_{1}, s_{2}, \ldots, s_{n}\right\}$ of observed consumption values and the estimation produced by forecasting algorithm $\widehat{S}=\left\{\widehat{s}_{1}, \widehat{s}_{2}, \ldots, \widehat{s}_{n}\right\}$, the MAPE is defined as:

$$
\operatorname{MAPE}(S, \widehat{S})=\frac{1}{n} \sum_{t=1}^{n}\left|\frac{s_{t}-\widehat{s}_{t}}{s_{t}}\right|
$$

The MAPE is an intuitive metric. However, it has a major drawback, i.e., it is not robust to the division by values approaching zero. Many households in the dataset have zero consumption on certain time slots, which makes the MAPE undefined, Furthermore, it is quite common to have households with very small consumption values, which makes the MAPE very large, approaching infinity.

Unlike the MAPE, the MAE and the RMSE do not suffer from the division by values approaching zero, since the MAE is defined as

$$
\operatorname{MAE}(S, \widehat{S})=\frac{1}{n} \sum_{t=1}^{n}\left(s_{t}-\widehat{s}_{t}\right)
$$

and the RMSE is defined as

\footnotetext{
${ }^{1}$ http://www.ucd.ie/issda/data/commissionforenergyregulationcer/
}

$$
\operatorname{RMSE}(S, \widehat{S})=\sqrt{\frac{1}{n} \sum_{t=1}^{n}\left(s_{t}-\widehat{s}_{t}\right)^{2}}
$$

However, they are scale-dependent metrics. Since the average hourly consumption of households in the dataset varies between $0.05 \mathrm{kWh}$ and $3.83 \mathrm{kWh}$, we need scale-independent metrics to aggregate the forecasting error of these different households. Moreover, scale-independent metrics can be useful to compare not only the forecasting error of different households, but also the forecasting error of different temporal aggregations or consumer groups. ${ }^{2}$

To this end, we suggest to use other metrics that are both, scale-independent, and robust to the division by values approaching zero, namely the Normalized Mean Absolute Error (NMAE) and the Normalized Root Mean Square Error (NRMSE). The NMAE is defined as

$$
\operatorname{NMAE}(S, \widehat{S})=\frac{\operatorname{MAE}(S, \widehat{S})}{\|S\|_{1}}=\frac{\sum_{t=1}^{n}\left|s_{t}-\widehat{s}_{t}\right|}{\sum_{t=1}^{n}\left|s_{t}\right|},
$$

and the NRMSE is defined as

$$
\operatorname{NRMSE}(S, \widehat{S})=\frac{\operatorname{RMSE}(S, \widehat{S})}{\|S\|_{2}}=\sqrt{\frac{\sum_{t=1}^{n}\left(s_{t}-\widehat{s}_{t}\right)^{2}}{\sum_{t=1}^{n} s_{t}^{2}}} .
$$

While one zero measurement is enough to make the MAPE undefined (or approaches infinity), all measurements need to be zero to make the NMAE or the NRMSE undefined.

\section{ForecAsting A LARGE NUMBER OF HouseHOLdS}

\section{A. Features}

There are two important challenges in selecting features for residential electricity load forecasting. First, different houses might have different energy consumption behavior. Thus, features that are relevant to one house might not be relevant to other houses. Second, we have a large number of houses. Therefore, feature selection should be done automatically.

To solve both challenges, we first build a (large) feature universe and then apply a feature selection algorithm to select features that are relevant to each house. We consider both, historical load and contextual features. To forecast the load at time (or hour) $t$, for 1 hour ahead forecasting, we consider the historical load data from time $t-1$ to $t-336$, i.e., $\left\{s_{t-1}, s_{t_{2}}, \ldots, s_{t-336}\right\} .{ }^{3}$ While for 24 hour ahead forecasting, we consider the historical load data from time $t-24$ to $t-336$ (since the historical load data from time $t-1$ to $t-23$ is not available in this case).

The CER dataset does not contain any information about the house or the persons who live in the house. Thus, for contextual features, we consider day of week, hour of day, and weather information. Since there is no information about the city/location of each house, we crawl the historical weather

\footnotetext{
${ }^{2}$ Apart from MAPE, MAE, and RMSE, there are also other options, such as the adjusted error [13]. See Section V for the discussion about the adjusted error.

${ }^{3}$ Of course, longer time duration can also be considered here, in the price of memory and computation cost.
} 


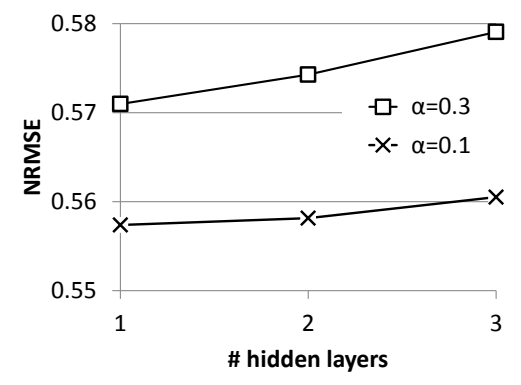

Fig. 1: MLP model evaluation (using NRMSE) using different number of hidden layers and learning rates $\alpha$ on randomly chosen 25 households. The lower the better. In the end, we use one hidden layer and $\alpha=0.1$.

data of the three biggest cities in Ireland, i.e., Dublin, Cork, and Limerick. ${ }^{4}$ We use 48 hours historical temperature and humidity data, ${ }^{5}$ from time $t-1$ to $t-48$ for 1 hour ahead forecasting, and from time $t-24$ to $t-71$ for 24 hour ahead forecasting. Additionally, we also include the mean and the median of those three cities to the feature set.

Up to this point, our feature universe contains approximately 800 variables. Next, we apply Correlation-based Feature Selection (CFS) to each house. This method selects subset of features that are highly correlated with the response variable while having low inter-correlation between each other [14]. As a result, we obtain a (much) reduced subset of relevant features for each house.

\section{B. Learning Algorithms}

Various learning algorithms have been used to forecast large-scale electricity demand. Recent literature suggests Support Vector Regression (SVR) as one of the most effective models to forecast future energy consumption [9], [32]. Other well established methods are Linear Regression and MultiLayer Perceptron (MLP). In this section, we briefly describe our model setup.

1) Linear Regression configuration: A linear model to predict the load at time $t$ is defined as:

$$
y=\boldsymbol{\theta}^{T} \boldsymbol{x}+\epsilon
$$

where $\boldsymbol{\theta}$ is the vector of coefficients, $\boldsymbol{x}$ is the feature vector, and $\epsilon$ is the error term. We estimate the coefficients and the error term of the linear model using ridge regression (other methods, of course, can also be used).

2) MLP configuration: We use one hidden layer with sigmoid activation functions. The output can be written as $y=\boldsymbol{W}_{2} \times \Theta\left(\boldsymbol{W}_{1} \cdot \boldsymbol{x}+\boldsymbol{B}_{1}\right)+\boldsymbol{B}_{2}$ where $\boldsymbol{x}$ is the input vector, $y$ is the output value, $\boldsymbol{W}_{1}, \boldsymbol{W}_{2}, \boldsymbol{B}_{1}$, and $\boldsymbol{B}_{2}$ are the coefficient matrices, and $\Theta$ is the sigmoid operator. Each component $x_{j}$ of the input vector $\boldsymbol{x}$ is standardized, i.e., $x_{j}^{*}=\left(x_{j}-\mu_{j}\right) / \sigma_{j}$, where $\mu_{j}$ is the mean and $\sigma_{j}$ is the standard deviation of the values in the $j$ th dimension. To avoid overfitting, a validation

\footnotetext{
${ }^{4}$ We obtained the weather-related data from http://www.wunderground.com.

${ }^{5}$ Apart from temperature, humidity has also been used in real-world implementation to forecast electricity demand. See, e.g., [22].
}

\begin{tabular}{|c|c|c|c|c|}
\hline$C \backslash \gamma$ & 0 & 0.01 & 0.1 & 1 \\
\hline $1 . E+00$ & 0.69 & 0.62 & 0.59 & 0.57 \\
\hline $1 . E+01$ & 0.62 & 0.59 & 0.58 & 0.57 \\
\hline $1 . E+02$ & 0.59 & $\mathbf{0 . 5 8}$ & 0.57 & 0.58 \\
\hline $1 . E+03$ & 0.58 & 0.58 & 0.57 & 0.63 \\
\hline $1 . E+04$ & 0.58 & 0.57 & 0.58 & 0.82 \\
\hline $1 . E+05$ & 0.57 & 0.57 & & \\
\hline
\end{tabular}

(a)

\begin{tabular}{|c|c|c|c|c|}
\hline$C \backslash \gamma$ & 0 & 0.01 & 0.1 & 1 \\
\hline $1 . E+00$ & 0.02 & 0.03 & 0.02 & 0.02 \\
\hline $1 . E+01$ & 0.03 & 0.02 & 0.02 & 0.20 \\
\hline $1 . E+02$ & 0.02 & $\mathbf{0 . 0 2}$ & 0.11 & 0.02 \\
\hline $1 . E+03$ & 0.02 & 0.02 & 0.02 & 0.02 \\
\hline $1 . E+04$ & 0.02 & 0.02 & 0.02 & 0.04 \\
\hline $1 . E+05$ & 0.02 & 0.11 & & \\
\hline
\end{tabular}

(b)

\begin{tabular}{|c|c|c|c|c|}
\hline$C \backslash \gamma$ & 0 & 0.01 & 0.1 & 1 \\
\hline $1 . E+00$ & 2.1 & 2.7 & 3.2 & 4.6 \\
\hline $1 . E+01$ & 2.3 & 2.6 & 3.1 & 3.2 \\
\hline $1 . E+02$ & 2.3 & 2.4 & 4.0 & 8.1 \\
\hline $1 . E+03$ & 2.7 & 3.0 & 8.9 & 45 \\
\hline $1 . E+04$ & 4.0 & 8.2 & 50 & 222 \\
\hline $1 . E+05$ & 7.2 & 52 & & \\
\hline
\end{tabular}

(c)

Fig. 2: SVR model evaluation for individual forecasting on the randomly chosen 25 households: (a) average NRMSE on the validation set given different $C$ and $\gamma$, (b) standard deviation on the average, (c) average running time. The lower the better. In the end, we choose $C=100$ and $\gamma=0.01$. While there are some other settings which yield better NRMSE, they typically require considerably longer running time.

set is constructed by randomly selecting $30 \%$ of the instances in the training set. The coefficient matrices are learnt using gradient descent, with learning rate of $\alpha=0.1$ (see the evaluation of different hidden layers and learning rates in Figure 1). The stopping criterion is triggered when the error on the validation set (calculated after each epoch) has increased 20 times in a row.

3) SVR configuration: SVR is a regression method based on Support Vector Machine (SVM) that has been developed in 1996 by Vapnik (see also the tutorial by Smola and Schlkopf [33]). In this work, we use the SVR implementation provided by the LIBSVM library developed by Chang and Lin [7].

SVR must be provided with the SVM error cost $C$ and a kernel function. For the kernel function, we use the RBF kernel, similar to [9]. Next, to find suitable values for $C$ and $\gamma$, we split the training set into two parts: a sub-training set and a validation set. The SVR is trained on the sub-training set, and evaluated on the validation set. For $C$ we test a set of values $\left\{1,10,10^{2}, 10^{3}, 10^{4}, 10^{5}\right\}$, while for $\gamma$ we test a set of values $\{0,0.01,0.1,1\}$.

For individual load forecasting, we find that different values of $C$ and $\gamma$ do not result in significant NRMSE differences (see Figure $2 \mathrm{a}$ and $2 \mathrm{~b}$ ) . However, they strongly affect the computation time, which dramatically increase when $C \geq 1000$ or $\gamma \geq 0.1$ (see Figure 2c). Thus, for individual forecast, we use $C=100$ and $\gamma=0.01$. On the other hand, for aggregate forecast, different settings of $C$ and $\gamma$ result in significant differences in terms of NRMSE (see Figure 3). We found that $C=1000$ and $\gamma=1$ is the best setting.

\section{Results}

In addition to features and learning algorithms, we also explore $p$ th root transformation. That is, instead of modeling 


\begin{tabular}{|c|c|c|c|c|c|}
\hline$C \backslash \gamma$ & 0.001 & 0.01 & 0.1 & 1 & 10 \\
\hline $1 . E-01$ & 0.418 & 0.415 & 0.395 & 0.356 & 0.318 \\
\hline $1 . E+00$ & 0.415 & 0.392 & 0.226 & 0.160 & 0.201 \\
\hline $1 . E+01$ & 0.391 & 0.200 & 0.074 & 0.070 & 0.082 \\
\hline $1 . E+02$ & 0.197 & 0.073 & 0.064 & 0.052 & 0.082 \\
\hline $1 . E+03$ & 0.073 & 0.066 & 0.059 & 0.045 & 0.065 \\
\hline $1 . E+04$ & 0.066 & 0.065 & 0.054 & 0.045 & 0.072 \\
\hline $1 . E+05$ & 0.066 & 0.061 & 0.050 & 0.050 & \\
\hline $1 . E+06$ & 0.065 & 0.057 & 0.047 & 0.063 & \\
\hline $1 . E+07$ & 0.061 & 0.054 & 0.046 & 0.087 & \\
\hline $1 . E+08$ & 0.063 & 0.110 & 0.188 & 0.149 & \\
\hline
\end{tabular}

Fig. 3: SVR model evaluation (measured by average NRMSE) for aggregate forecasting. The lower the better.

the response variable $\left(s_{t}\right)$ as is, we model its $p$ th root $\left(s_{t}^{1 / p}\right)$, and then transform the forecasted value back to its original dimension by raising it to the $p$ th power $\left(\left(\widehat{s}_{t}\right)^{p}\right)$. Since the distribution of household energy consumption are skewed to the left toward zero, $p$ th root transformation could help to make it more normal and easier to model.

As a comparison to the three learning algorithms described in Section III, we also employ Seasonal ARIMA for both, 1 hour and 24 hour ahead forecasting. Before using Seasonal ARIMA, however, we need to properly identify the order of the autoregressive, integrated, and moving average terms (for both, the seasonal and non-seasonal parts). Similar to the challenges that we face in the feature selection procedure, there are two important challenges here. First, since different households might have different energy consumption behavior, we need to identify the right orders for each household (i.e., the orders that are suitable for one household might not be suitable for others). Second, we have a large number of households. Thus, the identification procedure need to be done automatically. To this end, we apply the stepwise model space exploration algorithm outlined in [20] to each household. Starting from a small set of models, the algorithm iteratively explore the "neighbors" of the best model found so far. The algorithm stops when it cannot find a model better than the current best model.

Tables I and II show the performance of Seasonal Arima (SARIMA) using the setting described above, and Linear Regression (LR), Multi-Layer Perceptron (MLP), and Support Vector Regression (SVR) using the setting described in Section III-A and III-B. Both tables show that the $p$ th root transformation mostly improves the NMAE of the models. The tables also show that, in this case, the three learning algorithms LR, MLP, and SVR significantly outperform SARIMA.

\section{Cluster-BAsed Aggregate Forecasting}

In order to provide an aggregate forecast of a set of individually-monitored households, it is possible to define two extreme strategies: (1) aggregate the energy consumption of all households into one time series (the aggregate consumption), then forecast the aggregate consumption, and (2) forecast the energy consumption of each household separately, then aggregate the forecasts. Since the patterns in aggregate consumption are more regular than that of individual consumption (see also Figure 4), intuitively, strategy (1) should outperform strategy
TABLE I: Average NRMSE and NMAE (with its 95\% confidence interval) of SARIMA, LR, MLP, SVR for 1 hour ahead load forecasting at the level of the individual customer. Root transformation $\left(s_{t}{ }^{1 / p}\right.$, with $\left.p>1\right)$ can be used to improve NMAE.

\begin{tabular}{|c|c|c|c|c|}
\hline & & $p=1$ & $p=2$ & $p=4$ \\
\hline \multirow{4}{*}{ 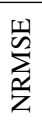 } & SARIMA & $0.582 \pm 0.007$ & $0.582 \pm 0.007$ & $0.589 \pm 0.007$ \\
\hline & LR & $\mathbf{0 . 5 5 7} \pm \mathbf{0 . 0 0 7}$ & $0.562 \pm 0.007$ & $0.571 \pm 0.007$ \\
\hline & MLP & $0.575 \pm 0.008$ & $0.569 \pm 0.007$ & $0.578 \pm 0.008$ \\
\hline & SVR & $0.573 \pm 0.008$ & $0.571 \pm 0.007$ & $0.572 \pm 0.007$ \\
\hline \multirow{4}{*}{$\sum_{Z}^{L}$} & SARIMA & $0.534 \pm 0.009$ & $0.485 \pm 0.008$ & $0.478 \pm 0.007$ \\
\hline & LR & $0.495 \pm 0.009$ & $0.461 \pm 0.007$ & $0.456 \pm 0.007$ \\
\hline & MLP & $0.535 \pm 0.014$ & $0.477 \pm 0.009$ & $0.468 \pm 0.008$ \\
\hline & SVR & $0.461 \pm 0.007$ & $0.448 \pm 0.007$ & $0.452 \pm 0.007$ \\
\hline
\end{tabular}

TABLE II: Average NRMSE and NMAE (with its 95\% confidence interval) of SARIMA, LR, MLP, SVR for 24 hour ahead load forecasting at the level of the individual customer. Root transformation $\left(s_{t}{ }^{1 / p}\right.$, with $\left.p>1\right)$ can be used to improve NMAE.

\begin{tabular}{|c|c|c|c|c|}
\hline & & $p=1$ & $p=2$ & $p=4$ \\
\hline \multirow{4}{*}{ 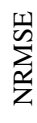 } & SARIMA & $0.671 \pm 0.007$ & $0.674 \pm 0.007$ & $0.688 \pm 0.008$ \\
\hline & LR & $\mathbf{0 . 6 0 7} \pm \mathbf{0 . 0 0 7}$ & $0.613 \pm 0.007$ & $0.623 \pm 0.008$ \\
\hline & MLP & $0.633 \pm 0.008$ & $0.630 \pm 0.008$ & $0.638 \pm 0.008$ \\
\hline & SVR & $0.628 \pm 0.008$ & $0.628 \pm 0.008$ & $0.629 \pm 0.008$ \\
\hline \multirow{4}{*}{$\sum_{Z}$} & SARIMA & $0.658 \pm 0.011$ & $0.598 \pm 0.009$ & $0.588 \pm 0.008$ \\
\hline & LR & $0.555 \pm 0.010$ & $0.515 \pm 0.008$ & $0.507 \pm 0.008$ \\
\hline & MLP & $0.601 \pm 0.016$ & $0.541 \pm 0.010$ & $0.527 \pm 0.009$ \\
\hline & SVR & $0.512 \pm 0.008$ & $0.501 \pm \mathbf{0 . 0 0 8}$ & $0.508 \pm 0.008$ \\
\hline
\end{tabular}

(2). Figure 5 shows clearly that the forecasting error decreases as the aggregation size increases.

In this section, we evaluate an alternative strategy (3), where we segment the households into $k$ clusters, aggregate the energy consumption of the households in each cluster, forecast

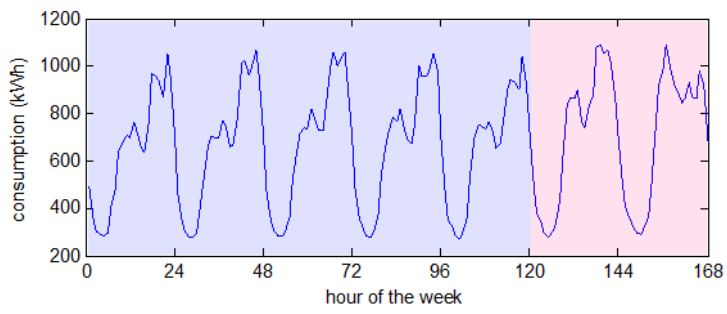

(a) Aggregate consumption (782 households).

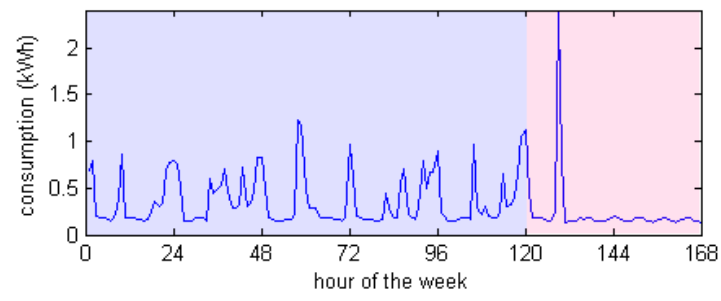

(b) Example consumption of a household (id 1002).

Fig. 4: A sample of hourly energy consumption from the CER dataset, from Monday, 2009-09-07 to Sunday, 2009-09-13. 


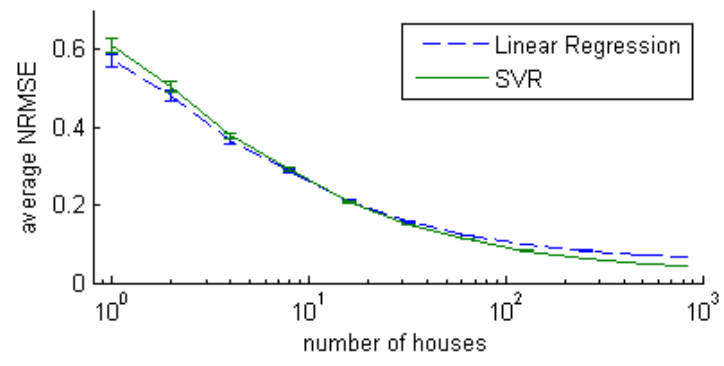

Fig. 5: The NRMSE of LR and SVR for 1 hour ahead forecasting (the lower the better). Forecasting error decreases as the aggregation size increases.

each cluster separately, and finally aggregate the $k$ forecasts into one aggregate forecast. Strategy (1) and (2) can also be seen as some special cases of strategy (3), where $k=1$ and $k=N=$ total customers, respectively. We refer to strategy (3) as the Cluster-based Aggregate Forecasting (CBAF). See Figure 6 for an illustration. The contributions of this section are: (i) we provide clustering algorithms to form clusters with some predefined/targeted characteristics (see Section IV-A), whereas previous works offer only little interpretation to the characteristics of the resulting clusters, (ii) we find that the improvement provided by the CBAF strategy depends not only on the number of clusters, but also on the size of the customer base (see Section IV-B).

\section{A. Clustering algorithms}

In order to investigate the effectiveness of CBAF, we define several clustering methods with clear objective, targeting a specific property of the resulting clusters:

- Max-AC: This method aims to maximize the autocorrelation of the energy consumption of the clusters. More specifically, this method uses the greedy clustering technique proposed in Algorithm 1 to find clusters such that the auto-correlation of the load of each cluster is maximized. Let $a c(S)$ be the average autocorrelation (up to a certain lag) of time series $S .{ }^{6}$ In addition, we define a cluster as a set of customers, and $S_{c}$ as the aggregate consumption time series of cluster $c$. Then this method uses Algorithm 1 by defining

$$
\Phi(c, x)=a c\left(S_{c \cup\{x\}}\right)-a c\left(S_{c}\right),
$$

where $x$ is a customer. As a consequence, customer $x$ is assigned to a cluster where $x$ provides the highest improvement to the auto-correlation of the clusters' energy consumption.

- Min-Stdev: This method aims to minimize the fluctuation in the clusters' energy consumption, which often becomes the main challenge to predict. In particular, it aims to minimize the standard deviation of the clusters' energy consumption. Let $s d(S)$ be the standard deviation of time series $S$. As in the Max-AC case, we define a cluster as a set of customers, and $S_{c}$

\footnotetext{
${ }^{6}$ In our implementation, we compute the auto-correlation up to lag 168 (or, 1 week preceding the target time). Other lags, however, can also be used.
}

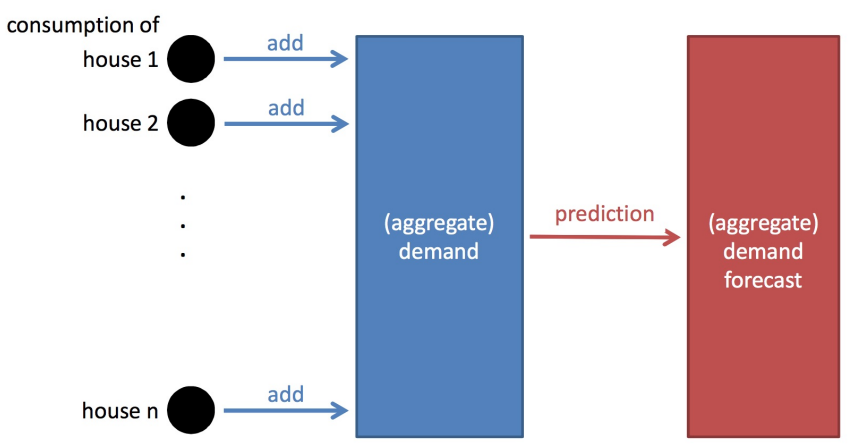

(a) Strategy 1: aggregate the energy consumption of all households into one time series (aggregate demand), then forecast the aggregate consumption.

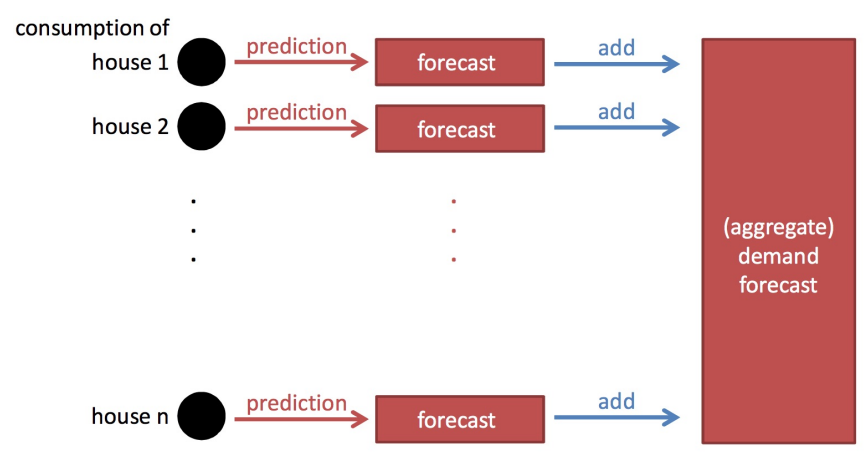

(b) Strategy 2: forecast the energy consumption of each household separately, then aggregate the forecasts.

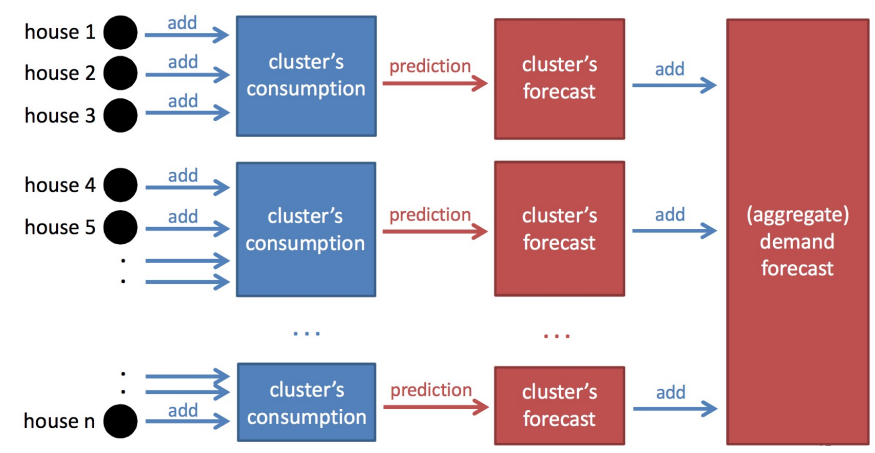

(c) Strategy 3: segment the households into $k$ clusters, aggregate the energy consumption of households in each cluster, forecast the demand of each cluster separately, and finally aggregate the $k$ forecasts into one aggregate forecast.

Fig. 6: Three strategies made available by smart meter data to forecast the aggregate demand of residential customers.

as the aggregate consumption time series of cluster $c$. Then this method uses Algorithm 1 by defining

$$
\Phi(c, x)=\left(s d\left(S_{c}\right)-s d\left(S_{c \cup\{x\}}\right)\right) \cdot|c|,
$$

where $x$ is a customer. As a consequence, customer $x$ is assigned to a cluster where $x$ minimizes the standard deviation of the cluster's aggregate consumption. Note that in the evaluation function $\Phi$, we multiply the standard deviation difference by $|c|$ so as to have a weighted difference, with respect to the size of the original cluster $c$ (before $x$ is added).

- Max-Sim: This method aims to maximize the similarity among customers within a cluster. Unlike previous 


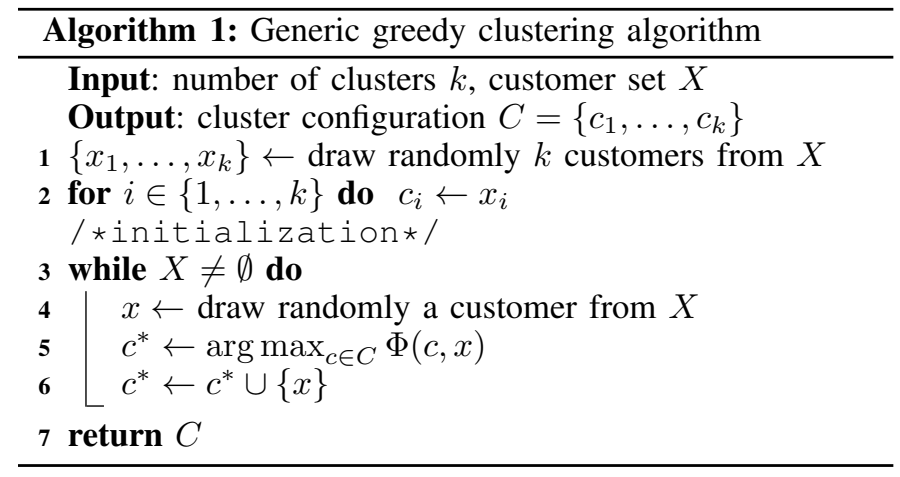

two methods, here we apply KMeans clustering algorithm to customer's 24-hour load profiles, where each hour is characterized by the distribution (or histogram) of the amount of energy consumed in that hour. More specifically, for each hour, we define a feature vector of length 21 . For the first 20 elements, the $i$ th element is the frequency of consumption between $(i-1) \times 0.5 \mathrm{kWh}$ and $i \times 0.5 \mathrm{kWh}$. The $21^{\text {st }}$ element is the frequency of consumption greater than $10 \mathrm{kWh}$. Finally, we apply KMeans on the customers' feature vectors, where each feature vector of a customer is of length $24 \times 21=504$.

- Random: Each customer is randomly assigned to any of the clusters with the equal probability.

In this experiment, we enlarge our dataset to include all residential customers who have no missing values. This results in the selection of 3,639 customers. Figures 7 shows the NRMSE, the NMAE and the MAPE of LR, MLP and SVR, for a different number of clusters $k$. When $k=1$, all customers are aggregated into a single cluster and a single prediction is performed. As $k$ increases, more clusters are created ( $k$ clusters to be precise), and the consumption of each cluster is forecasted separately. The forecasts are then aggregated into a single aggregate forecast. Note that, $k=1$ represents strategy (1), $k=N=3639$ strategy (2), and $1<k<N$ the CBAF strategy, which is the focus of this section. We do not show the forecasting result beyond $k=128$ as the error continues to increase beyond that of $k=1$. This fact clearly shows that, strategy (1) outperforms strategy (2). Additionally, there are some $1<k<N$ for which the forecasting error is lower than that of $k=1$. This fact confirms that CBAF indeed can be used to improve the accuracy of aggregate forecasting.

Interestingly, all clustering methods that we introduced (including Random) seem to be able to provide a lower forecasting error than that of strategy (1). Although in some cases Max-AC provides the lowest error curve as it aims to maximize the auto-correlation of the energy consumption within the clusters, ${ }^{7}$ the accuracies of these clustering methods are often marginally different. Therefore, choosing one clustering method against the others (or implementing the CBAF strategy) in a real-world scenario needs a more careful analysis, in the sense that we need to consider whether the advantage brought by a particular clustering method is greater than the

\footnotetext{
${ }^{7}$ Time series with higher auto-correlation is typically easier to forecast since it shows greater relationship between the current and the past values.
}

cost of implementing it.

\section{B. The Impact of the Size of the Customer Base}

Hitherto one might think that the improvement obtained by CBAF depends on the number of clusters, $k$. While this insight has been confirmed by Figure 7 , there is more to it than that since it turned out that the size of the customer base also plays an important role in the improvement. We repeat the experiments using different sized customer bases: 500, 1,000 , and 2,000 (drawn randomly from the original dataset of 3,639 customers). Note that, for the same $k$, a different size of customer bases implies different cluster sizes.

Figure 8 shows the improvement gained by SVR when we perform CBAF on different size of customer bases. While there is almost no positive improvement in the case of 500 customers (no matter which clustering method is used), some improvement may be noticed in the case of 1,000 customers or more. In general, the improvement increases with the size of the customer base.

If we assume that a "good" forecast models the true observation and a white noise (zero mean and finite variance), then it means that combining several good forecasts from several clusters into one aggregate forecast could neutralize the white noise. Thus, there is a trade-off between the size and the number of clusters. The size of the clusters should be big enough for the algorithm to deliver a reasonably good prediction, ${ }^{8}$ but not too big that there are not enough clusters (hence, predictions) to cancel out the noise.

In addition, since the number of clusters, $k$, strongly influence the cluster size, one might wonder whether it is possible to set a priori the best value for $k$. Because characteristics of a customer base vary from one to another, the right $k$ should be determined using cross validation on the training set. Apart from that, the experiment suggests that the improvement offered by CBAF will increase further as we incorporate more and more customers (due to the possibility to increase both, the number and the size of the clusters).

\section{RELATED WORK}

Electricity demand forecasting has been widely studied in the literature. In addition to studies that focusing on the forecasting methodology, ${ }^{9}$ researchers have also studied particular geographical areas or countries [2], [5], [6], [26], [29], [31]. Competitions have also been organized [9], [17]. All of them, however, focused on demand forecasting on a large scale, either at the regional or national level.

Due to the recent deployment of smart meters, forecasting energy demand at the residential level is a relatively new area. The work by Ghofrani et al. [12] can be considered as one of the earliest works in the field, where they forecast the electricity demand of a single household, using one day of training and one day of test data. Since then, some interesting results have been published. Tidemann et al., for example,

\footnotetext{
${ }^{8}$ See also Figure 5 about the relation between forecast accuracy and customer aggregation size.

${ }^{9}$ We have mentioned the references in Section I, i.e., Linear Regression [16], [30], ARMA [18], [34], Generalized Additive Models [4], [10], [41], Neural Networks [3], [15], [23] and Support Vector Regression [9], [32].
} 

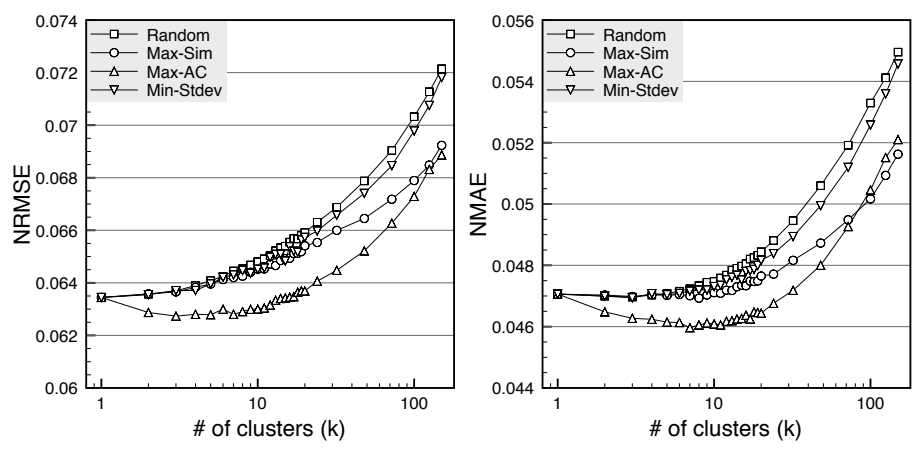

(a) LR
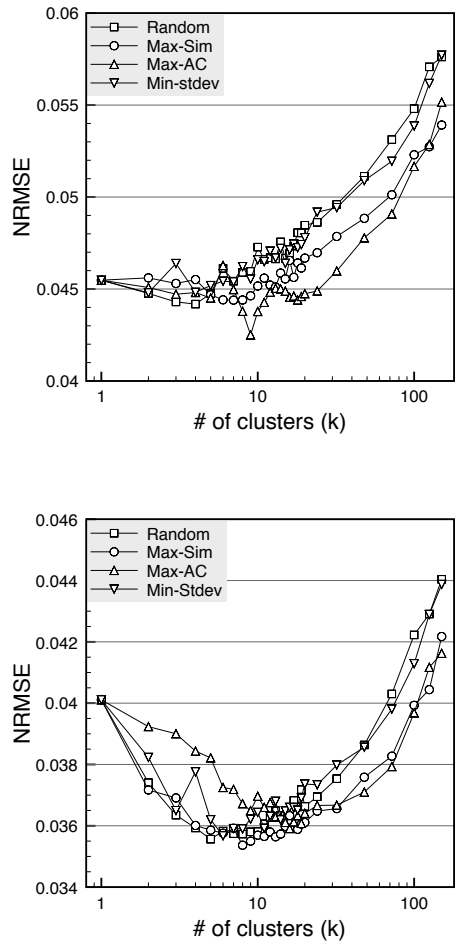

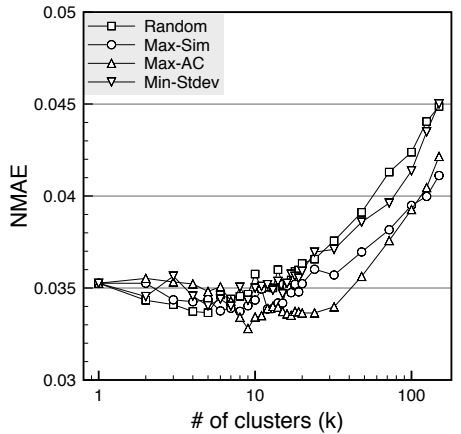

(b) MLP

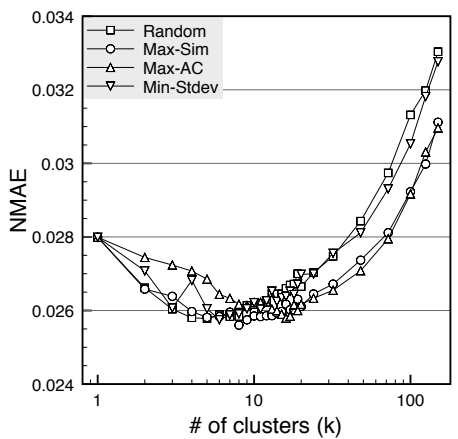

(c) SVR
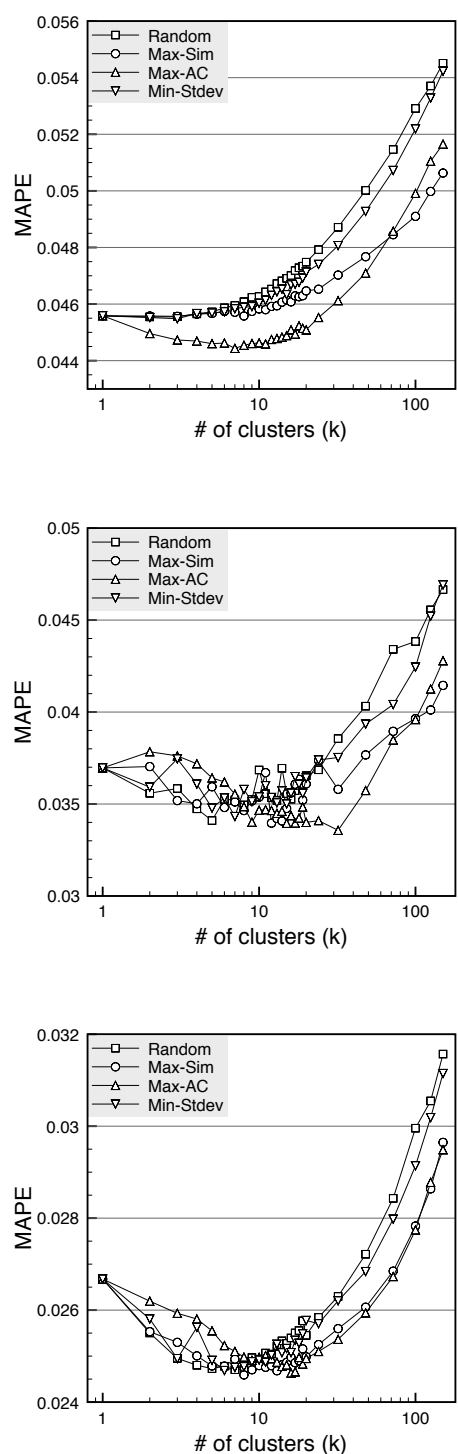

Fig. 7: The NRMSE, the NMAE and the MAPE for a different number of clusters $k$ (the lower the better). Total number of customers, $N=3639$. The best accuracy is obtained when $1<k<3639$, which shows the effectiveness of CBAF.

showed that due to irregularities in electricity demand at the household level, forecasting demand at the household level is indeed more difficult than at the distribution or transmission level [36]. Chaouch used functional wavelet-kernel and then improved it by clustering daily load curves and trained each cluster separately [8]. The approach took only the historical load curve as input, and therefore a careful modification need to be performed to account for external factors, such as calendar variables or temperatures. In contrast, by using machine learning algorithms (such as Linear Regression, Multi-Layer Perceptron, or Support Vector Machine), incorporating new external factors is essentially adding new elements to the feature vector. Thus, as there will be more and more contextual data concerning households available in the future, machine learning algorithms facilitate the seamless addition of new features. ${ }^{10}$

Haben et al. proposed the adjusted error measure to tolerate forecasted values that are slightly misplaced in time [13]. The measure can also be seen as a generalization of the standard p-norm error. More specifically, when the tolerance magnitude, $w$, is equal to zero, the measure reduces to the standard p-norm errors. It is not scale-independent, however, which makes it unsuitable to compare or aggregate the accuracy of the demand forecasts of different households. Furthermore, it requires permutation of the forecasts, and thus needs cubic time to compute, whereas most evaluation metrics takes only linear time.

Misiti et al. [25] and Alzate and Sinn [1] have also consid-

\footnotetext{
${ }^{10}$ Several works have used demographic information to estimate electricity demand. See, e.g., [21], [24], [26], [39].
} 


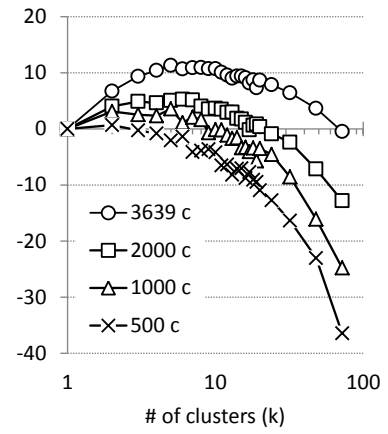

(a) Random

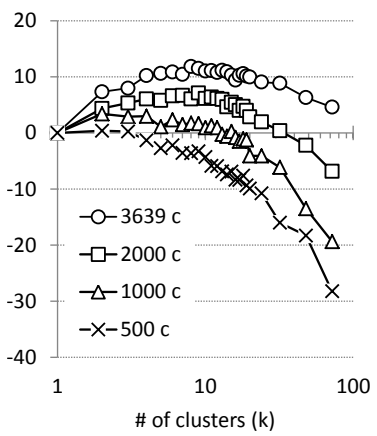

(b) $\mathrm{Max}-\mathrm{Sim}$

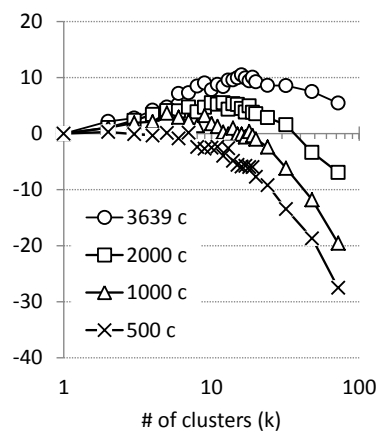

(c) $\mathrm{Max}-\mathrm{AC}$

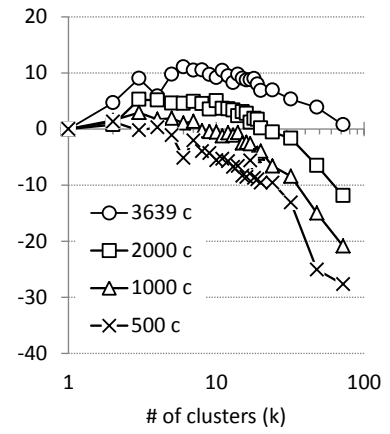

(d) Min-Stdev

Fig. 8: Percentage improvement in the NRMSE of the CBAF strategy (compared to the traditional aggregate forecast, $k=1$ ) of $500,1,000,2,000$, and 3,639 customers over a different number of clusters and clustering methods (the higher the better). The larger the customer set, the higher the improvement gained by CBAF.

ered CBAF. ${ }^{11}$ However, Misiti et al. consider only industrial customers, and Alzate and Sinn consider a mix of residential customers and small/medium enterprises. Additionally, they focused on wavelet-based and kernel spectral clustering ${ }^{12}$ and did not investigate the effect of different customer sizes to the improvement provided by CBAF.

\section{CONCLUSION}

In this paper we evaluated various machine learning algorithms for short-term individual and aggregate forecasting (1 hour and 24 hour ahead) of residential electricity consumption. Additionally, to measure the forecasting accuracy at the household level effectively, we use evaluation metrics that are scaleindependent and robust to values approaching zero, namely the NRMSE and the NMAE. Individual forecasting, in general, is a challenging task (with NRMSE around 0.5-0.6 and NMAE around $0.4-0.5)$. Aggregate forecasting, however, have better accuracy (with NRMSE around 0.04 and NMAE around 0.03).

Additionally, in our setting, LR, MLP, and SVR outperformed SARIMA in the 1 hour and 24 hour ahead forecasting of individual households. Although MLP and SVR are more sophisticated than LR, in individual forecasting, their forecasting performances are not significantly better than LR (especially with $p$ th root transformation, where $p=2$ or $p=4$ ). In aggregate forecasting, however, SVR is significantly better than LR (see, e.g, Figure 7 where $k=1$ ). Therefore, in a realworld scenario, one should consider the trade-off between the advantage brought by a more sophisticated model and the cost to implement and maintain it.

In addition, we proposed a generic algorithm to segment customers according to a predefined/targeted objective. We showed its usefulness by forming clusters that (1) maximize the auto-correlation and (2) minimize the standard deviation of the clusters' energy consumption. When using CBAF, empirically we found that clustering customers into 8 to 10 clusters delivers the best forecasting accuracy across different

\footnotetext{
${ }^{11}$ They refer to CBAF as disaggregated load forecasting.

${ }^{12}$ Interestingly, although [1], [25] and our work focus on different customer types and use different forecasting and clustering algorithms, all conclude that clustering customers and forecasting each cluster separately could indeed improve aggregate forecasts.
}

error metrics, forecating algorithms, and clustering approaches. Additionally, we also found that the improvement provided by the CBAF strategy depends not only on the number of clusters, but also on the size of customer base. More specifically, CBAF improves traditional aggregate forecasting when the size of the customer base is above a certain threshold. Conversely, no improvement is achieved when the size of the customer base is below this threshold, no matter which clustering methods is applied. In general, however, the larger the size of the customer base, the higher the improvement offered by CBAF.

Finally, the effectiveness of CBAF shall not be limited only to smart grid analytics. Practically, it can be used in other fields as well, where multiple sensor measurements are available for building an aggregate view of an environment. For example, in the field of participatory sensing or weather forecasting, one can used CBAF similarly by first clustering sensor nodes, forecasting them separately, and then aggregate the forecasts. Additionally, one might be more interested to aggregate by averaging rather than summing them up, e.g., for obtaining a more accurate temperature of a certain region from multiple sensors.

\section{ACKNOWLEDGMENT}

The authors would like to thank Alhussein Fawzi and the anonymous reviewers for their helpful comments and discussion. The research leading to these results has received funding from the European Union's Seventh Framework Programme (FP7/2007-2013) under grant agreement no. 288322 (Wattalyst) and 288021 (EINS). Tri Kurniawan Wijaya is supported in part by an IBM PhD Fellowship award.

\section{REFERENCES}

[1] C. Alzate and M. Sinn, "Improved electricity load forecasting via kernel spectral clustering of smart meters," in IEEE 13th International Conference on Data Mining (ICDM), Dec 2013, pp. 943-948.

[2] H. A. Amarawickrama and L. C. Hunt, "Electricity demand for Sri Lanka: A time series analysis," Energy, vol. 33, no. 5, pp. 724 - 739, 2008.

[3] N. An, W. Zhao, J. Wang, D. Shang, and E. Zhao, "Using multi-output feedforward neural network with empirical mode decomposition based signal filtering for electricity demand forecasting," Energy, vol. 49, no. 0 , pp. $279-288,2013$. 
[4] A. Ba, M. Sinn, Y. Goude, and P. Pompey, "Adaptive learning of smoothing functions: Application to electricity load forecasting," in NIPS, P. L. Bartlett, F. C. N. Pereira, C. J. C. Burges, L. Bottou, and K. Q. Weinberger, Eds., 2012, pp. 2519-2527.

[5] V. Bianco, O. Manca, and S. Nardini, "Electricity consumption forecasting in Italy using linear regression models," Energy, vol. 34, no. 9, pp. 1413 - 1421, 2009.

[6] J. R. Cancelo, A. Espasa, and R. Grafe, "Forecasting the electricity load from one day to one week ahead for the Spanish system operator," International Journal of Forecasting, vol. 24, no. 4, pp. 588 - 602, 2008.

[7] C.-C. Chang and C.-J. Lin, "LIBSVM: A library for support vector machines," ACM Transactions on Intelligent Systems and Technology, vol. 2, pp. 27:1-27:27, 2011.

[8] M. Chaouch, "Clustering-based improvement of nonparametric functional time series forecasting: Application to intra-day household-level load curves," IEEE Transactions on Smart Grid, vol. 5, no. 1, pp. 411419, Jan 2014.

[9] B.-J. Chen, M.-W. Chang et al., "Load forecasting using support vector machines: A study on EUNITE competition 2001," IEEE Transactions on Power Systems, vol. 19, no. 4, pp. 1821-1830, 2004.

[10] S. Fan and R. Hyndman, "Short-term load forecasting based on a semi-parametric additive model," IEEE Transactions on Power Systems, vol. 27, no. 1, pp. 134-141, Feb 2012.

[11] C. W. Gellings, The Smart Grid: Enabling energy efficiency and demand response. The Fairmont Press, Inc., 2009.

[12] M. Ghofrani, M. Hassanzadeh, M. Etezadi-Amoli, and M. Fadali, "Smart meter based short-term load forecasting for residential customers," in North American Power Symposium (NAPS), Aug 2011, pp. $1-5$.

[13] S. Haben, J. Ward, D. V. Greetham, C. Singleton, and P. Grindrod, "A new error measure for forecasts of household-level, high resolution electrical energy consumption," International Journal of Forecasting, vol. 30 , no. 2, pp. $246-256,2014$.

[14] M. A. Hall, "Correlation-based feature selection for machine learning," Ph.D. dissertation, The University of Waikato, 1999.

[15] H. S. Hippert, C. E. Pedreira, and R. C. Souza, "Neural networks for short-term load forecasting: A review and evaluation," IEEE Transactions on Power Systems, vol. 16, no. 1, pp. 44-55, 2001.

[16] T. Hong, "Short-term electric load forecasting," Ph.D. dissertation, North Carolina State University, Sep. 2010.

[17] T. Hong, P. Pinson, and S. Fan, "Global energy forecasting competition 2012," International Journal of Forecasting, vol. 30, no. 2, pp. 357 363, 2014.

[18] S.-J. Huang and K.-R. Shih, "Short-term load forecasting via ARMA model identification including non-gaussian process considerations," IEEE Transactions on Power Systems, vol. 18, no. 2, pp. 673-679, May 2003.

[19] S. Humeau, T. K. Wijaya, M. Vasirani, and K. Aberer, "Electricity load forecasting for residential customers: Exploiting aggregation and correlation between households," in Sustainable Internet and ICT for Sustainability (SustainIT), 2013, Oct 2013, pp. 1-6.

[20] R. J. Hyndman and Y. Khandakar, "Automatic time series forecasting: The forecast package for R," Journal of Statistical Software, vol. 27, no. 3, pp. 1-22, 72008

[21] A. Jarrah Nezhad, T. K. Wijaya, M. Vasirani, and K. Aberer, "SmartD: Smart Meter Data Analytics Dashboard," in The 5th ACM International Conference on Future Energy Systems (e-Energy'14), 2014.

[22] "PJM Empirical Analysis of Demand Response Baseline Methods," White Paper, KEMA, Inc., Apr. 2011
[23] A. Khotanzad, R. Afkhami-Rohani, T.-L. Lu, A. Abaye, M. Davis, and D. Maratukulam, "ANNSTLF-A Neural-Network-based Electric Load Forecasting System," IEEE Transactions on Neural Networks, vol. 8, no. 4, pp. 835-846, Jul 1997.

[24] J. Z. Kolter and J. Ferreira, "A large-scale study on predicting and contextualizing building energy usage," in $A A A I$, W. Burgard and D. Roth, Eds. AAAI Press, 2011.

[25] M. Misiti, Y. Misiti, G. Oppenheim, and J.-M. Poggi, "Optimized clusters for disaggregated electricity load forecasting," REVSTAT, vol. 8, pp. 105-124, 2010.

[26] Z. Mohamed and P. Bodger, "Forecasting electricity consumption in New Zealand using economic and demographic variables," Energy, vol. 30 , no. 10 , pp. $1833-1843,2005$.

[27] A. Mohd, E. Ortjohann, A. Schmelter, N. Hamsic, and D. Morton, "Challenges in integrating distributed energy storage systems into future Smart Grid," in IEEE International Symposium on Industrial Electronics, June 2008, pp. 1627-1632.

[28] K. Moslehi and R. Kumar, "A reliability perspective of the Smart Grid," IEEE Transactions on Smart Grid, vol. 1, no. 1, pp. 57-64, June 2010.

[29] H.-T. Pao, "Comparing linear and nonlinear forecasts for Taiwan's electricity consumption," Energy, vol. 31, no. 12, pp. 2129 - 2141, 2006.

[30] A. Papalexopoulos and T. Hesterberg, "A regression-based approach to short-term system load forecasting," IEEE Transactions on Power Systems, vol. 5, no. 4, pp. 1535-1547, Nov 1990.

[31] S. Saab, E. Badr, and G. Nasr, "Univariate modeling and forecasting of energy consumption: the case of electricity in Lebanon," Energy, vol. 26, no. 1, pp. $1-14,2001$.

[32] N. Sapankevych and R. Sankar, "Time series prediction using support vector machines: A survey," IEEE Computational Intelligence Magazine, vol. 4, no. 2, pp. 24 -38, May 2009.

[33] A. J. Smola and B. Schölkopf, "A tutorial on support vector regression," Statistics and Computing, vol. 14, no. 3, pp. 199-222, Aug. 2004.

[34] J. W. Taylor, "Triple seasonal methods for short-term electricity demand forecasting," European Journal of Operational Research, vol. 204, no. 1, pp. 139-152, 2010.

[35] The Commission for Energy Regulation (CER), "Electricity Smart Metering Customer Behaviour Trials Findings Report," Tech. Rep., May 2011

[36] A. Tidemann, B. A. Høverstad, H. Langseth, and P. Öztürk, "Effects of scale on load prediction algorithms," in 22nd International Conference on Electricity Distribution, 2013.

[37] T. K. Wijaya, M. Vasirani, and K. Aberer, "When bias matters: An economic assessment of demand response baselines for residential customers," IEEE Transactions on Smart Grid, vol. 5, no. 4, pp. 17551763, July 2014

[38] T. K. Wijaya, "Pervasive Data Analytics for Sustainable Energy Systems," Ph.D. dissertation, EPFL, Lausanne, 2015.

[39] T. K. Wijaya, T. Ganu, D. Chakraborty, K. Aberer, and D. P. Seetharam, "Consumer segmentation and knowledge extraction from smart meter and survey data," in SDM, M. J. Zaki, Z. Obradovic, P.-N. Tan, A. Banerjee, C. Kamath, and S. Parthasarathy, Eds. SIAM, 2014, pp. $226-234$

[40] T. K. Wijaya, T. G. Papaioannou, X. Liu, and K. Aberer, "Effective consumption scheduling for demand-side management in the smart grid using non-uniform participation rate," in Sustainable Internet and ICT for Sustainability (SustainIT), 2013. IEEE, 2013, pp. 1-8.

[41] T. K. Wijaya, M. Sinn, and B. Chen, "Forecasting uncertainty in electricity demand," in AAAI-15 Workshop on Computational Sustainability, 2015. 